\title{
Correlation quantum beats induced by non-Markovian effect
}

Received: date / Accepted: date

\begin{abstract}
For two qubits independently coupled to their respective structured reservoirs (Lorentzian spectrum), quantum beats for entanglement and discord are found which are the result of quantum interference between correlation oscillations induced by local non-Markovian environments. We also discuss the preservation of quantum correlations by the effective suppression of the spontaneous emission.
\end{abstract}

Keywords Quantum beat · Entanglement · Quantum discord · NonMarkovian effect

\section{Introduction}

Quantum entanglement and discord, two different measures of quantum correlation, have been receiving much attention recent years. Interest that people pour on entanglement lies in mainly two aspects. One is the important role of entanglement in quantum computation and quantum information processing [1, such as quantum teleportation, quantum dense coding, quantum cryptography, remote quantum-state preparation [2], quantum remote control [3], and distributed quantum computation [4, etc. The other lies in the understanding of nature of non-locality in quantum mechanics $[5$. Though quantum entanglement not necessarily leads to non-locality, quantum non-locality does indeed arise from measurement correlations on quantum entangled states. Quantum discord, a relatively new concept born at the turn of this century, is regarded as the measure of all nonclassical correlations in a bipartite system [6], being the entanglement a particular case of it. As shown in [7,8, deterministic quantum computation with one pure qubit may be carried out in the situation of no entanglement but discord. In addition, quantum discord is also used 
in studies of quantum phase transition [9, 10, estimation of quantum correlations in the Grover search algorithm [11, and to characterize the class of initial system-bath states for which the quantum dynamics is equivalent to a completely positive map [12.

Realistic quantum systems cannot avoid interactions with their environments, which can alter the properties of quantum correlation. In the last decade, the influences of Markovian environments on quantum entanglement have been investigated extensively [13. An interesting phenomenon, named "entanglement sudden death" (ESD) [14 for a pair of entangled qubits exposed to local Markovian environments, was found, which triggered reams of related works. Different from entanglement, quantum discord in similar conditions decays only in asymptotic time [15, which signifies that quantum discord is more robust against Markovian noise than entanglement.

More recently, much attention and interest have been devoted to the study of non-Markovian dynamics of open systems [16, 17, 18, 19, 20, 21,22. As people have found that many relevant open systems, such as quantum optical system [23], quantum dot 24], color-core spin in semiconductor [25], as well as quantum chemistry [26] and biological systems [27, require to be treated in non-Markovian dynamics. The properties of entanglement evolution for a pair of entangled qubits exposed to local non-Markovian environments have been studied already 28, 29, 30, 31. Entanglement trapping in engineering structured environment has been proposed. In this paper, we further investigate the time-evolution properties of quantum correlation (including entanglement and discord) in this physical system. We find that a single non-Markovian environment only induces correlation oscillation, but the cooperation of two local non-Markovian environments can lead to quantum interference between correlation oscillations. As a result, an interesting physical phenomenon-correlation quantum beat-is observed. We will also highlight the mechanism for the preservation of quantum correlations in the considered system.

The paper is organized as follows. In Sec. 2, we present the exact dynamics for a two-qubit system coupled to local non-Markovian environments. And in Secs. 3 and 4, we study the quantum beats for entanglement and discord respectively for the studied open system. Sec. 5 is devoted to the problem of preservation of quantum correlations for the considered system. A conclusion is arranged in Sec. 6.

\section{Non-Markovian dynamics}

In this part, we present the exact dynamics of the open quantum system considered in this paper. We consider two noninteracting qubits $A$ and $B$, each of them locally coupled to a vacuum reservoir via Jaynes-Cummings model,

$$
H=\omega_{0} \sigma_{+} \sigma_{-}+\sum_{k} \omega_{k} b_{k}^{+} b_{k}+\sum_{k}\left(g_{k} \sigma_{+} b_{k}+\text { h.c. }\right) .
$$

Here $\omega_{0}$ is the transition frequency of qubit and $\sigma_{ \pm}$are the corresponding raising and lowering operators, while the index $k$ labels the field modes of 
the reservoir with frequencies $\omega_{k}$, creation and annihilation operators $b_{k}^{+}, b_{k}$ and coupling constants $g_{k}$. Eq. (1) is one of the most fundamental systemenvironment interaction models which is extensively used in modeling dynamics of open quantum systems $[21,23,28,32$. According to the method presented in 28, the dynamics of the two-qubit system can be obtained simply from the one of the individual qubits. The exact dynamics of each qubit can be described as 32,

$$
\begin{aligned}
& \rho_{11}(t)=|G(t)|^{2} \rho_{11}(0) \\
& \rho_{00}(t)=\rho_{00}(0)+\left[1-|G(t)|^{2}\right] \rho_{11}(0), \\
& \rho_{10}(t)=G(t) \rho_{10}(0) \\
& \rho_{01}(t)=G^{*}(t) \rho_{01}(0)
\end{aligned}
$$

Where $\rho_{i j}=\langle i|\rho| j\rangle$ with $|0\rangle$ and $|1\rangle$ being ground and excited states of qubit. The function $G(t)$ is defined as the solution of the integro-differential equation,

$$
\frac{d}{d t} G(t)=-\int_{0}^{t} d t_{1} f\left(t-t_{1}\right) G\left(t_{1}\right)
$$

with initial condition $G(0)=1$. The kernel $f\left(t-t_{1}\right)$ denotes the two-point reservoir correlation function which is the Fourier transformation of the spectral density $J(\omega)$,

$$
f\left(t-t_{1}\right)=\int d \omega J(\omega) \exp \left[i\left(\omega_{0}-\omega\right)\left(t-t_{1}\right)\right],
$$

with $\omega_{0}$ being the transition frequency of qubit.

Although the process applies to any form of initial correlation state of two-qubit system, for simplicity, we only consider two types of initial Bell-like states,

$$
|\psi\rangle=a|00\rangle+b e^{i \theta}|11\rangle
$$

and

$$
|\phi\rangle=\alpha|01\rangle+\beta e^{i \delta}|10\rangle .
$$

Where $a, b, \alpha, \beta, \theta, \delta$ are real and normalized as $a^{2}+b^{2}=1$ and $\alpha^{2}+\beta^{2}=1$. Owing to the influence of the environment, the coherence of the system will vanish asymptotically. Thus, the state of the system at any time $t$ would be a mixed state. Under the product basis $\{|00\rangle,|01\rangle,|10\rangle,|11\rangle\} \equiv\{|1\rangle,|2\rangle,|3\rangle,|4\rangle\}$, the dynamical state of the two-qubit system can be written in the form $\rho(t)=$ $\left[\rho_{i j}(t)\right]$ with $i, j=1,2,3,4$. By the method presented in [28, combined with the dynamical evolution of eq.(2) for single open qubit, we find that the density matrix of the open two-qubit system only has the following nonzero elements,

$$
\begin{aligned}
& \rho_{11}(t)=a^{2}+\left(1-\left|G_{A}\right|^{2}\right)\left(1-\left|G_{B}\right|^{2}\right) b^{2} \\
& \rho_{22}(t)=\left(1-\left|G_{A}\right|^{2}\right)\left|G_{B}\right|^{2} b^{2} \\
& \rho_{33}(t)=\left|G_{A}\right|^{2}\left(1-\left|G_{B}\right|^{2}\right) b^{2} \\
& \rho_{44}(t)=\left|G_{A}\right|^{2}\left|G_{B}\right|^{2} b^{2} \\
& \rho_{14}(t)=G_{A}^{*} G_{B}^{*} a b e^{-i \theta} \\
& \rho_{41}(t)=G_{A} G_{B} a b e^{i \theta}
\end{aligned}
$$


for initial state $|\psi\rangle$, and

$$
\begin{aligned}
& \rho_{11}(t)=\left(1-\left|G_{B}\right|^{2}\right) \alpha^{2}+\left(1-\left|G_{A}\right|^{2}\right) \beta^{2}, \\
& \rho_{22}(t)=\alpha^{2}\left|G_{B}\right|^{2}, \\
& \rho_{33}(t)=\beta^{2}\left|G_{A}\right|^{2}, \\
& \rho_{23}(t)=\alpha \beta e^{-i \delta} G_{A}^{*} G_{B}, \\
& \rho_{32}(t)=\alpha \beta e^{i \delta} G_{A} G_{B}^{*},
\end{aligned}
$$

for initial state $|\phi\rangle$. All other matrix elements are zero. Here we use $G_{A} \equiv$ $G_{A}(t)$ and $G_{B} \equiv G_{B}(t)$ to denote the $G(t)$ function for qubits $A$ and $B$ respectively, which are determined by eq.(3). In the following sections, we will study the dynamical properties of quantum correlations based on these equations.

\section{Entanglement quantum beat}

Using the results presented in above section, one can study the evolution of quantum entanglement for given initial states. We use Wootters concurrence 33 as the measure of quantum entanglement. For a two-qubit system with density matrix $\rho$, the concurrence $C$ is defined as

$$
C=\max \left\{0, \lambda_{1}-\lambda_{2}-\lambda_{3}-\lambda_{4}\right\} .
$$

Here $\lambda_{1} \geq \lambda_{2} \geq \lambda_{3} \geq \lambda_{4}$ are the square roots of the eigenvalues of the matrix $R=\rho \widetilde{\rho}$, with $\widetilde{\rho}=\sigma_{y}^{(1)} \otimes \sigma_{y}^{(2)} \rho^{*} \sigma_{y}^{(1)} \otimes \sigma_{y}^{(2)}$ and the sign "*" standing for complex conjugate. For the states given by eqs.(7) and (8), the corresponding concurrences are

$$
C_{\psi}(t)=\max \left\{0,2\left|G_{A} G_{B} a b\right|-2 b^{2}\left|G_{A} G_{B}\right| \sqrt{\left(1-\left|G_{A}\right|^{2}\right)\left(1-\left|G_{B}\right|^{2}\right)}\right\}
$$

and

$$
C_{\phi}(t)=2\left|\alpha \beta G_{A} G_{B}\right|,
$$

respectively. The notable characteristic is that the dynamical entanglement is independent of the phase angles $\theta, \delta$ in the initial Bell-like states. In addition, the evolution of state $|\psi\rangle$ can happen entanglement sudden death (ESD) when the condition $|a|-|b| \sqrt{\left(1-\left|G_{A}\right|^{2}\right)\left(1-\left|G_{B}\right|^{2}\right)}<0$ is fulfilled.

The results of eqs. (10) and (11) apply to, in general, any structured environment. Here we assume that two identical qubits $A$ and $B$ (having equal transition frequency $\omega_{0}$ and free decay rate $\gamma_{0}$ ) are respectively plug into different imperfect optical cavities, so that the spectrum may be characterized by the Lorentzian distribution,

$$
J_{j}(\omega)=\frac{\gamma_{0} \lambda^{2}}{2 \pi\left[\left(\omega_{0}-\omega-\Delta_{j}\right)^{2}+\lambda^{2}\right]} .
$$

Where $\Delta_{j}=\omega_{0}-\omega_{c j}$ with $j=A, B$ is the frequency detuning between qubit $j$ and the corresponding cavity-mode $\omega_{c j}$. We assume that the two cavities have 
equal photon-leakage rate $\lambda$. For this Lorentzian spectrum, the correlation function in eq.(3) can be easily evaluated as

$$
f_{j}\left(t-t_{1}\right)=\frac{1}{2} \gamma_{0} \lambda e^{-\left(\lambda-i \Delta_{j}\right)\left(t-t_{1}\right)},
$$

and thus the function $G(t)$ is

$$
G_{j}(t)=e^{-\left(\lambda-i \Delta_{j}\right) t / 2}\left[\cosh \left(\frac{d_{j} t}{2}\right)+\frac{\lambda-i \Delta_{j}}{d} \sinh \left(\frac{d_{j} t}{2}\right)\right],
$$

with $d_{j}=\sqrt{\left(\lambda-i \Delta_{j}\right)^{2}-2 \gamma_{0} \lambda}$. Inserting this expression into eqs.(10) and (11), we can obtain the dynamical entanglement for the given initial states $|\psi\rangle$ and $|\phi\rangle$.

For the Lorentzian spectrum, two typical coupling regimes-weak and strong regimes-can be distinguished customarily. For the weak coupling regime, $\gamma_{0}<$ $\lambda / 2$, the correlation time $\tau_{B} \simeq \lambda^{-1}$ of reservoir is smaller than the relaxation time $\tau_{R} \simeq \gamma_{0}^{-1}$ of the qubit. In this regime, the qubit dynamics behaves like essentially Markovianity and the quantum entanglement between qubits $A$ and $B$ reduces monotonously. In contrast, for the strong coupling regime, $\gamma_{0}>\lambda / 2$, the correlation time of reservoir is greater than or of the same order as the relaxation time of the qubit. In this case, the non-Markovian effect may cause the quantum entanglement between the two qubits to rise, or even to form entanglement oscillations. In particular, we find that when the detunings $\Delta_{A}$ and $\Delta_{B}$ are both large and have tiny difference, then the time evolution for the concurrences given by eqs.(10) and (11) will happen quantum beat, an interesting and fundamental quantum phenomenon encountered in quantum optics. We show this in Fig.1, where the initial Bell states are chosen, but for other non-maximally entangled states, similar phenomenon of quantum beats may also exist. It is worthwhile to point out that these quantum beats originate purely from non-Markovian effect, as no any direct or mediated interaction exists between the two qubits. This is different from the case [34] of two qubits coupled to a common cavity, where the two qubits can interact through common reservoir. In addition, Fig.1 also shows that the entanglement decay for state $|\psi\rangle$ is faster than that for state $|\phi\rangle$, which indicates that the later is more robust when exposing to the considered reservoirs. The physical reason is visible: In state $|\phi\rangle$, at most one of the qubits is in excited state so that the rate of spontaneous emission is smaller. While in state $|\psi\rangle$, two qubits can be simultaneously in excited state, leading to stronger spontaneous emission.

Physically, the entanglement quantum beat is the result of quantum interference of the dynamical entanglements. If only one of the qubits couples to environment and the other is immune from any noise, then the non-Markovian effect only leads the dynamical entanglement between the two qubits to oscillate but not to happen quantum beat. This features are shown in Fig.2 (top row), where the solid line denotes the evolution of concurrence when only qubit $A$ couples to environment with detuning $\Delta_{A}=50 \lambda$, and the dot line for the case where only qubit $B$ couples to environment with detuning $\Delta_{B}=45 \lambda$. The different detunings $\Delta_{A}$ and $\Delta_{B}$ leads to the tiny difference in frequencies for 

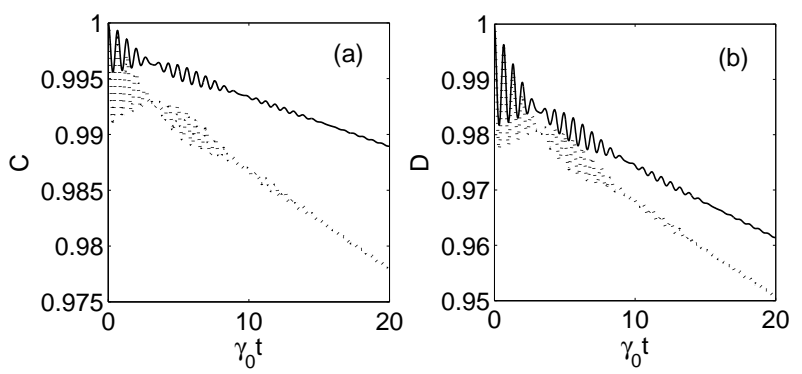

Fig. 1 Time evolution of concurrence for initial states $|\phi\rangle=1 / \sqrt{2}\left[|01\rangle+e^{i \delta}|10\rangle\right]$ (solid line), and $|\psi\rangle=1 / \sqrt{2}\left[|00\rangle+e^{i \theta}|11\rangle\right]$ (dot line). Where $\lambda=0.2 \gamma_{0}, \Delta_{A}=50 \lambda, \Delta_{B}=45 \lambda$.

the two corresponding entanglement oscillations. In this way, when the two qubits couple simultaneously to their local environments, the entanglement oscillations produce interference, leading to entanglement quantum beat. The phenomenon is similar to the coherence superposition of two Mechanical oscillations with tiny frequency difference to form classical beat, except that the total dynamical entanglement is not simply the sum of the two components of the dynamical entanglements depicted in Fig.2.

In the above discussion, we produce the tiny frequency difference for the two entanglement oscillations by different cavity frequencies $\omega_{c j}$. In fact, we may also realize the entanglement quantum beat through other parameter control, for example by adjusting the transition frequencies of the qubits or the Lorentzian widths $\lambda_{j}$ of the cavities. In this sense, the quantum beat may be used to indicate the differences of the cavity-mode frequencies, the qubit-transition frequencies or the cavity-decay rates, according to different situations.

\section{Quantum beat for discord}

Besides the entanglement quantum beat, we find that the time evolution of quantum discord also appears similar phenomenon in considered system. In order to demonstrate this, let us first briefly review the notion of quantum discord. For a bipartite quantum system, the quantum mutual information between the two subsystems $A$ and $B$ is given by

$$
\mathcal{I}\left(\rho_{A B}\right)=S\left(\rho_{A}\right)+S\left(\rho_{B}\right)-S\left(\rho_{A B}\right),
$$

where $S(\rho)=-\operatorname{Tr}(\rho \log \rho)$ is the von Neumann entropy of the density matrix $\rho$. The quantum mutual information has fundamental physical significance, and is usually used as a measure of total correlations that include quantum and classical ones. The classical correlation may be defined in terms of projective measurement. Suppose we perform a set of projective measurement $\left\{\Pi_{k}^{B}\right\}$ on the subsystem $B$, then the probability for measurement outcome $k$ may be reads 

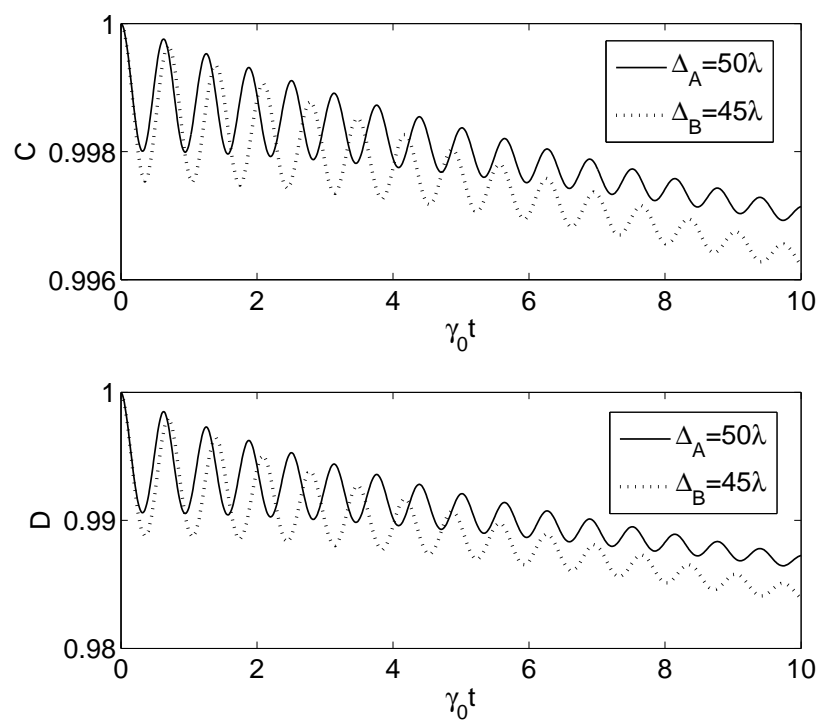

Fig. 2 Time evolution of concurrence (top row) and discord (bottom row) for initial state $|\phi\rangle=1 / \sqrt{2}\left[|01\rangle+e^{i \delta}|10\rangle\right]$. Where $\lambda=0.2 \gamma_{0}$.

$p_{k}=\operatorname{Tr}_{A B}\left[\left(I^{A} \otimes \Pi_{k}^{B}\right) \rho_{A B}\left(I^{A} \otimes \Pi_{k}^{B}\right)\right]$ with $I^{A}$ the identity operator for subsystem $A$. After this measurement, the state of subsystem $A$ is described by the conditional density operator $\rho_{A \mid k}=\operatorname{Tr}_{B}\left[\left(I^{A} \otimes \Pi_{k}^{B}\right) \rho_{A B}\left(I^{A} \otimes \Pi_{k}^{B}\right)\right] / p_{k}$. We define the upper limit of the difference between the von Neumann entropy $S\left(\rho_{A}\right)$ and the based-on-measurement quantum conditional entropy $\sum_{k} p_{k} S\left(\rho_{A \mid k}\right)$ of subsystem $A$, i.e.,

$$
\mathcal{Q}\left(\rho_{A B}\right)=\sup _{\left\{\Pi_{k}^{B}\right\}}\left[S\left(\rho_{A}\right)-\sum_{k} p_{k} S\left(\rho_{A \mid k}\right)\right],
$$

as the classical correlation of the two subsystems. The maximum is taken for all possible projective measurements. In the end, the quantum discord is defined as the difference between the total and classical correlations [6],

$$
D\left(\rho_{A B}\right)=\mathcal{I}\left(\rho_{A B}\right)-\mathcal{Q}\left(\rho_{A B}\right)
$$

Though in general the calculation of quantum discord is formidable, for $\mathrm{X}$ states of two-qubit system, an analytical method has been presented 35. Employing this method, a straightforward calculation gives the quantum discord of the state of eqs.(7) as,

$$
D_{\psi}(\rho)=S\left(\rho^{B}\right)+\sum_{j=0}^{3} \lambda_{j} \log _{2} \lambda_{j}+\min \left\{S_{1}, S_{2}\right\} .
$$



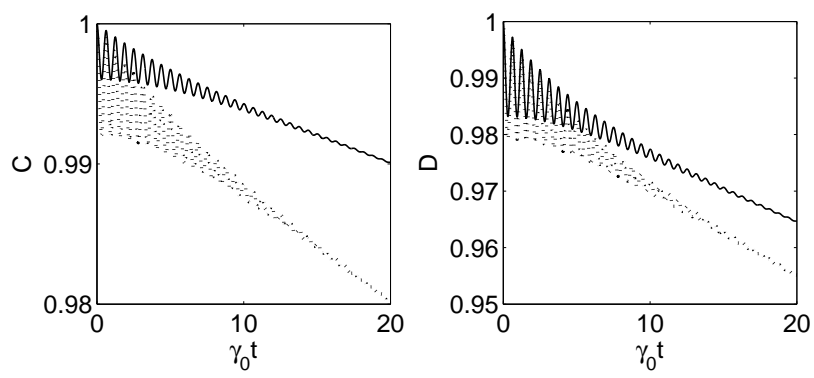

Fig. 3 Time evolution of quantum discord for initial states $|\phi\rangle=1 / \sqrt{2}\left[|01\rangle+e^{i \delta}|10\rangle\right]$ (solid line), and $|\psi\rangle=1 / \sqrt{2}\left[|00\rangle+e^{i \theta}|11\rangle\right]$ (dot line). Where $\lambda=0.2 \gamma_{0}, \Delta_{A}=50 \lambda, \Delta_{B}=45 \lambda$.

Where $S\left(\rho^{B}\right)=-\left[\left(\rho_{11}+\rho_{33}\right) \log _{2}\left(\rho_{11}+\rho_{33}\right)+\left(\rho_{22}+\rho_{44}\right) \log _{2}\left(\rho_{22}+\rho_{44}\right)\right]$ is the Von Neumann entropy of qubit $B$, given the two-qubit state described by eq. (7). And $\lambda_{0}=\frac{1}{2}\left[\left(\rho_{11}+\rho_{44}\right)+\sqrt{\left(\rho_{11}-\rho_{44}\right)^{2}+4\left|\rho_{14}\right|^{2}}\right], \lambda_{1}=\frac{1}{2}\left[\left(\rho_{11}+\rho_{44}\right)-\right.$ $\left.\sqrt{\left(\rho_{11}-\rho_{44}\right)^{2}+4\left|\rho_{14}\right|^{2}}\right], \lambda_{2}=\rho_{22}, \lambda_{3}=\rho_{33}$, are the eigenvalues of density matrix given by eq. (7). $S_{1}=\left(\rho_{22}+\rho_{44}\right) S\left(\rho_{0}\right)+\left(\rho_{11}+\rho_{33}\right) S\left(\rho_{1}\right)$ with $S\left(\rho_{0}\right)=$ $-\frac{1-\eta}{2} \log _{2} \frac{1-\eta}{2}-\frac{1+\eta}{2} \log _{2} \frac{1+\eta}{2}, S\left(\rho_{1}\right)=-\frac{1-\eta^{\prime}}{2} \log _{2} \frac{1-\eta^{\prime}}{2}-\frac{1+\eta^{\prime}}{2} \log _{2} \frac{1+\eta^{\prime}}{2}, \eta=$ $\left|\rho_{22}-\rho_{44}\right| /\left(\rho_{22}+\rho_{44}\right), \eta^{\prime}=\left|\rho_{11}-\rho_{33}\right| /\left(\rho_{11}+\rho_{33}\right)$. And $S_{2}=-\frac{1-\epsilon}{2} \log _{2} \frac{1-\epsilon}{2}-$ $\frac{1+\epsilon}{2} \log _{2} \frac{1+\epsilon}{2}$ with $\epsilon=\sqrt{\left(\rho_{11}+\rho_{22}-\rho_{33}-\rho_{44}\right)^{2}+4\left|\rho_{14}\right|^{2}}$.

Similarly, the quantum discord for the state of eq.(8) can be written as,

$$
D_{\phi}(\rho)=S\left(\rho^{B}\right)+\rho_{11} \log _{2} \rho_{11}+\left(\rho_{22}+\rho_{33}\right) \log _{2}\left(\rho_{22}+\rho_{33}\right)+\min \left\{S_{1}, S_{2}\right\}
$$

Here $S\left(\rho^{B}\right)=-\left[\left(\rho_{11}+\rho_{33}\right) \log _{2}\left(\rho_{11}+\rho_{33}\right)+\rho_{22} \log _{2} \rho_{22}\right]$ is Von Neumann entropy of qubit $B$, given the two-qubit state described by eq.(8). $S_{1}=\left(\rho_{11}+\right.$ $\left.\rho_{33}\right)\left[-\frac{1-\Lambda}{2} \log _{2} \frac{1-\Lambda}{2}-\frac{1+\Lambda}{2} \log _{2} \frac{1+\Lambda}{2}\right]$ with $\Lambda=\left|\rho_{11}-\rho_{33}\right| /\left(\rho_{11}+\rho_{33}\right)$. And $S_{2}=$ $-\frac{1-\Lambda^{\prime}}{2} \log _{2} \frac{1-\Lambda^{\prime}}{2}-\frac{1+\Lambda^{\prime}}{2} \log _{2} \frac{1+\Lambda^{\prime}}{2}$ with $\Lambda^{\prime}=\sqrt{\left(\rho_{11}+\rho_{22}-\rho_{33}\right)^{2}+4\left|\rho_{23}\right|^{2}}$.

In Fig.3, we show the time evolution of quantum discord according to eqs. (18) and (19). On the whole, this figure is similar to Fig.1. The discords for the given states decrease oscillatorily and the behavior of quantum beat is visible. This quantum beat is also purely from non-Markovian effect, because quantum discord for Markovian processes only reduces monotonously 15. The physical mechanism is also due to the quantum interference of discords: When only one of the qubits couples to environment, the quantum discords fall oscillatorily and no quantum beat occurs [ see Fig.2 (bottom row)]; But when the two qubits couple simultaneously to their local environments, the quantum interference lead to discord quantum beat. Also, the discord for state $|\psi\rangle$ decays faster than that for state $|\phi\rangle$, duo to the difference of decay rates of the two states. The visible difference between figs. 3 and 1 is that, in the same parameter conditions, discord decays more rapidly. 


\section{Protection of quantum correlation}

The protection of quantum correlation is of great importance to quantum information processing. Most works [28, 29,30, 31] have been done for the protection of entanglement in non-Markovian environments. Here, we mainly discuss the problem of preserving discord. It is well known that the radiative properties of an atom in a cavity will change fundamentally compared to in free space. Spontaneous emission may be decreased [36. when the atom is set detuning enough from the cavity. Especially, if the transition frequency of the atom lies below the fundamental frequency of the cavity, spontaneous emission is significantly inhibited. Conversely, if the atom is set to be resonant with the cavity, then the spontaneous emission will be quicken vastly [37,38.

The enhancement and suppression of spontaneous emission of atoms in cavities may be used to effectively manipulate quantum correlation, even in the non-Markovian environments. For example, to protect quantum correlation between two qubits, one can put the two qubits into two separate vacuum cavities, as implemented in the previous sections. By adjusting the cavity frequencies well mistuned from the qubit transitions, the spontaneous emission of the qubits is then inhibited and the quantum correlation between them is protected effectively. In Fig. 4, we depict the time evolution of discord between the two qubits, according to eqs.(18) and (19). [The evolution of concurrence is similar but decreases slower.] For simplicity, we set the two qubit to have equal detuning, $\Delta_{A}=\Delta_{B} \equiv \Delta$ [For different detunings, the quantum beat may occur as seen in previous sections]. One may see clearly from this figure that in the resonant regime, the discord decreases rapidly. However, for large detunings, the discord can be protected much effectively.

Two significant elements for the protection of quantum correlations in the above setup deserve to mention. One is the amount of frequency detuning between qubit and cavity mode. The larger the detuning is, the smaller the rate of spontaneous emission is, and the slower the quantum correlation reduces. This feature has been observed already from Fig.4. Another significant element is the monochromaticity of the cavity mode. For given qubit-cavity detuning, the well the monochromaticity of the cavity mode is, the smaller the rate of spontaneous emission is, and the slower the quantum correlation decreases. This feature is shown in Fig.5. [Note that the two features mentioned here are also true for concurrence.] Especially, in the limit that the cavity mode is completely monochromatic [which mean the Lorentzian width in eq.(12) $\lambda \rightarrow 0$ ] and for non-resonant case, one has $d_{j} \rightarrow i|\Delta|$ and $\left|G_{j}\right| \rightarrow 1$. Then eqs.(7) and (8) return respectively to their initial states of eqs. (5) and (6). This implies that qubits in non-leakage and non-resonant cavities, the spontaneous emissions are completely suppressed and their quantum states are preserved exactly.

At the end of this section, we point out that the protection of quantum correlations may be distinguished as two cases. In the first case, the quantum correlations in a given state decrease very slowly in time, so that in a relatively long time they remain on a very high level, though they may vanish eventually. 

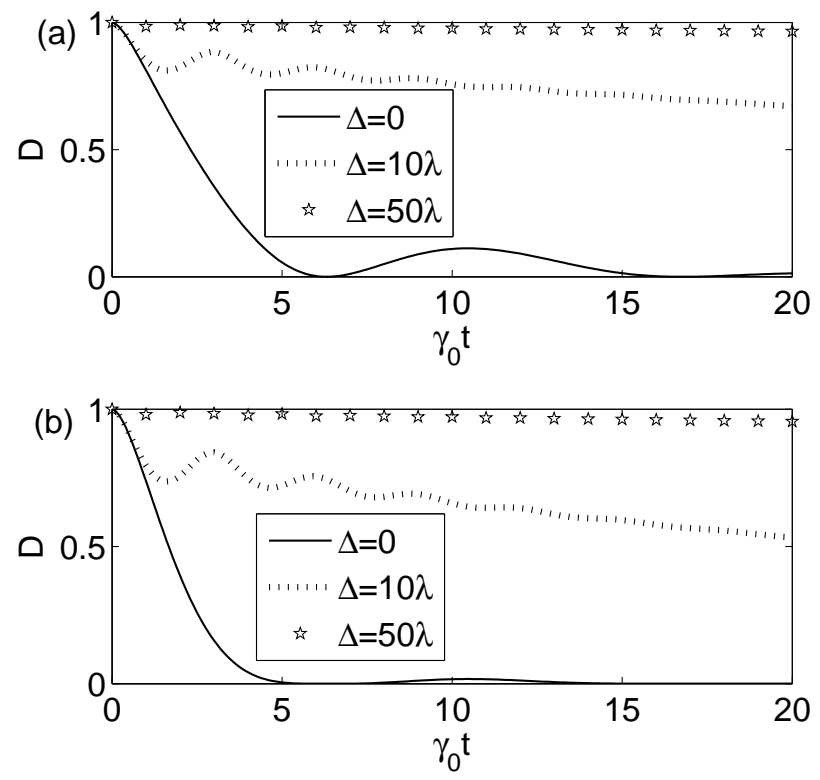

Fig. 4 Time evolution of quantum discord for different detunings $\Delta$ and for initial states: (a) $|\phi\rangle=1 / \sqrt{2}\left[|01\rangle+e^{i \delta}|10\rangle\right]$, and (b) $|\psi\rangle=1 / \sqrt{2}\left[|00\rangle+e^{i \theta}|11\rangle\right]$. Where $\lambda=0.2 \gamma_{0}$.
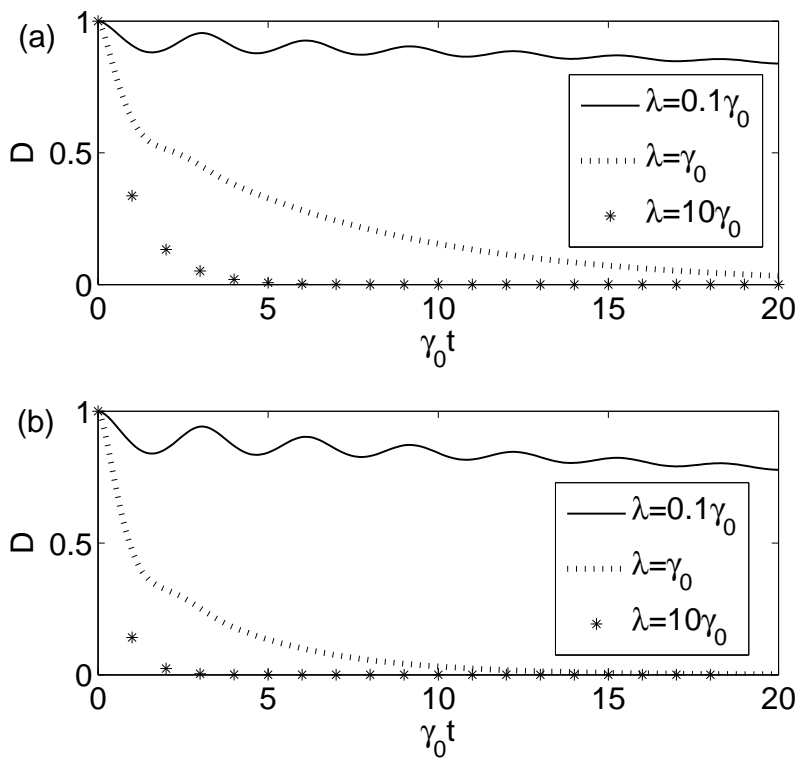

Fig. 5 Time evolution of quantum discord for different Lorentzian widths $\lambda$ and for initial states: (a) $|\phi\rangle=1 / \sqrt{2}\left[|01\rangle+e^{i \delta}|10\rangle\right]$, and (b) $|\psi\rangle=1 / \sqrt{2}\left[|00\rangle+e^{i \theta}|11\rangle\right]$. Where $\Delta=2 \gamma_{0}$. 
This protection for quantum correlations is usually necessary in practice. What we studied above belong to this case. In the second case, the system evolves to a steady state in which some residual quantum correlations exist even for infinitely long time, though the amount of the quantum correlations may not be very high. We may visually call the latter case the "correlation trapping". It is worthwhile to point out that though the phenomenon of "entanglement trapping" in the local non-Markovian environments has been found already 29. 30, the study about preservation of discord is not trivial, because quantum discord may has different behaviors, such as the robustness against noises [15. 39, and the distinct features at phase transition [9, 10, etc.

\section{Conclusion}

The exact non-Markovian dynamics of quantum-correlations (quantum entanglement and discord) for two qubits independently coupled to their respective structured reservoirs (Lorentzian spectrum) have been investigated. We have found, under some parameter conditions, that non-Markovian effect can lead to quantum correlation oscillations which can further interfere to form correlation quantum beats. This kind of quantum interference may be used to detect the difference of the qubit-transition frequencies or to indicate the difference of the local non-Markovian environments. We have also discussed the preservation of quantum correlations by the effective suppression of the spontaneous emission. Good monochromaticity of cavity mode, and large frequency detuning between qubit and cavity mode can help to protect quantum discord and entanglement.

The intrinsic character of non-Markovian processes with memory lies in the back flow of information from environment to the system, which compared to Markovian processes gives rise to various distinct dynamical traits, such as the changes of dissipative [23] or dephasing [40] rates, the correlation of photons emitted by an atom [41, and the non-continuously reduction 28] of quantum entanglement or discord, etc. In this paper, we have further found that the correlation oscillations induced by local non-Markovian environments can take place interference to present correlation quantum beats. This discovery reveals further the dynamical features of quantum correlations and non-Markovian processes.

The observation of correlation quantum beats requires to enter strong coupling regime $\left(\gamma_{0}>\lambda / 2\right)$ and under large detuning conditions $(\Delta>>\lambda)$. In recent experiments with quantum dot micropillars [42], the quality factor up to $1.65 \times 10^{4}$ has been reported and a linewidth $\lambda$ of $9.6 \mu \mathrm{eV}$ for the fundamental mode (resonant frequency $\omega_{c} / 2 \pi=1.31832 \mathrm{eV}$ ) has been reached. The linewidth $\gamma_{0}$ of dot exciton can be changed from about $1.5 \mu \mathrm{eV}$ at zero temperature to $3 \mathrm{meV}$ at room temperature [43. Thus the strong coupling condition could be well met by temperature control. The large detuning condition is easy to be realized. Noting that $50 \lambda=480 \mu \mathrm{eV}$ and the nearest spectator mode with frequency $1.32070 \mathrm{eV}$, thus one can make the dot transition frequency $\omega_{0}$ to be 
large detuning from the cavity fundamental mode while simultaneously be immune from the spectator mode. It also need to note that the entanglement evolution in non-Markovian environments was studied experimentally, and ESD and entanglement birth were observed 22. These achievements have paved the way to experimentally simulate the paradigmatic models of open quantum systems.

\section{References}

1. Nielsen, M. A., Chuang, I. L.: Quantum Computation and Quantum Information. Cambridge University Press, Cambridge (2000)

2. Berry, D. W., Sanders, B. C.: Optimal remote state preparation. Phys. Rev. Lett. 90, 057901 (pp.1-4) (2003)

3. Huelga, S. F., Vaccaro, J. A., Chefles, A., Plenio, M. B.: Quantum remote control: Teleportation of unitary operations. Phys. Rev. A 63, 042303 (pp.1-4) (2001)

4. Cirac, J. I., Ekert, A. K., Huelga, S. F., Macchiavello, C.: Distributed quantum computation over noisy channels. Phys. Rev. A 59, 4249-4254 (1999)

5. Einstein, A., Podolsky, B., Rosen, R.: Can quantum mechanical description of physical reality be considered complete?. Phys. Rev. A 47 777-780 (1935)

6. Ollivier, H., Zurek, W. H.: Quantum discord: A measure of the quantumness of correlations. Phys. Rev. Lett. 88, 017901 (pp.1-4) (2001)

7. Datta, A., Shaji, A., Caves, C. M.: Quantum discord and the power of one qubit. Phys Rev. Lett. 100, 050502 (pp.1-4) (2008)

8. Lanyon, B. P., Barbieri, M., Almeida, M. P., White, A. G.: Experimental quantum computing without entanglement. Phys. Rev. Lett. 101, 200501 (pp.1-4) (2008)

9. Dillenschneider, R.: Quantum discord and quantum phase transition in spin chains. Phys. Rev. B 78, 224413 (pp.1-7) (2008)

10. Sarandy, M. S.: Classical correlation and quantum discord in critical systems. Phys. Rev. A 80, 022108 (pp.1-9) (2009)

11. Cui, J., Fan, H.: Correlations in the Grover search. J. Phys. A: Math. Theor. 43045305 (2010)

12. Shabani, A., Lidar, D. A.: Vanishing quantum discord is necessary and sufficient for completely positive maps Phys. Rev. Lett. 102, 100402 (pp.1-4) (2009)

13. Ann, K., Jaeger, G.: Finite-time destruction of entanglement and non-locality by environmental influences. Found. Phys. 39, 790-828 (2009)

14. Yu, T., Eberly, J. H.: Finite-time disentanglement via spontaneous emission. Phys. Rev. Lett. 93, 140404 (pp.1-4) (2004)

15. Werlang, T., Souza, S., Fanchini, F. F., Villas Boas, C. J.: Robustness of quantum discord to sudden death. Phys. Rev. A 80, 024103 (pp.1-4) (2009)

16. Breuer, H. P., Laine, E. M., Piilo, J.: Measure for the degree of non-Markovian behavior of quantum processes in open systems. Phys. Rev. Lett. 103210401 (pp.1-4) (2009)

17. Rivas, A., Huelga, S. F., Plenio, M. B.: Entanglement and non-Markovianity of quantum evolutions. Phys. Rev. Lett. 105050403 (pp.1-4) (2010)

18. Wolf, M., Eisert, J., Cubitt, T. S., Cirac, J. I.: Assessing non-Markovian quantum dynamics. Phys. Rev. Lett. 101150402 (pp.1-4) (2008)

19. Dijkstra, A. G., Tanimura, Y.: Non-Markovian entanglement dynamics in the presence of system-bath coherence. Phys. Rev. Lett. 104250401 (pp.1-4) (2010)

20. Jing, J., Yu, T.: Non-Markovian relaxation of a three-level system: Quantum trajectory approach. Phys. Rev. Lett. 105240403 (pp.1-4) (2010)

21. Haikka, P., Maniscalco, S.: Non-Markovian dynamics of a damped driven two-state system. Phys. Rev. A 81052103 (pp.1-11) (2010)

22. Xu, J. S., Li, C. F., Gong, M., Zou, X. B., Shi, C. H., Chen, G., Guo, G. C.: Experimental demonstration of photonic entanglement collapse and revival. Phys. Rev. Lett. 104100502 (pp.1-4) (2010) 
23. Breuer, H. P., Petruccione, F.: The theory of open quantum systems. Oxford University Press, Oxford (2007)

24. Kubota, Y., Nobusada, K.: Applicability of site-basis time-evolution equation for thermalization of exciton states in a quantum dot array. J. Phys. Soc. Jpn. 78114603 (pp.1-7) (2009)

25. Kane, B. E.: A silicon-based nuclear spin quantum computer. Nature 393 133-137 (1998)

26. Shao, J.: Decoupling quantum dissipation interaction via stochastic fields. J. Chem.

Phys. 1205053 (pp.1-4) (2004)

27. Chin, A. W., Datta, A., Caruso, F., Huelga, S. F., Plenio, M. B.: Noise-assisted energy transfer in quantum networks and light-harvesting complexes. New J. Phys.12 065002 (pp.1-16) (2010)

28. Bellomo, B., Lo Franco, R., Compagno, G.: Non-Markovian effects on the dynamics of entanglement. Phys. Rev. Lett. 99160502 (pp.1-4) (2007)

29. Bellomo, B., Lo Franco, R., Maniscalco, S., Compagno, G.: Entanglement trapping in structured environments. Phys. Rev. A 78 060302(R) (pp.1-4) (2008)

30. Tong, Q. J., An, J. H., Luo, H. G., Oh, C. H.: Mechanism of entanglement preservation. Phys. Rev. A 81052330 (pp.1-5) (2010)

31. Xiao, X., Fang, M. F., Li, Y. L., Zeng, K., Wu, C.: Robust entanglement preserving by detuning in non-Markovian regime. J. Phys. B: At. Mol. Opt. Phys. 42235502 (pp.1-6) (2009)

32. Laine, E. M., Piilo, J., Breuer, H. P.: Measure for the non-Markovianity of quantum processes. Phys. Rev. A 81062115 (pp.1-8) (2010)

33. Wootters, W. K.: Entanglement of formation of an arbitrary state of two qubits. Phys. Rev. Lett. 80, 2245-2248 (1998)

34. Francica, F., Maniscalco, S., Piilo, J., Plastina, F., Suominen, K. A.: Off-resonant entanglement generation in a lossy cavity. Phys. Rev. A 79032310 (pp.1-10) (2009)

35. Ali, M., Rau, A. R. P., Alber, G.: Quantum discord for two-qubit X states. Phys. Rev. A 81042105 (pp.1-7) (2010)

36. Kleppner, D.: u(3)-Boson model of nuclear collective motion. Phys. Rev. Lett. 47 223$226(1981)$

37. Goy, P., Raimond, J. M., Gross, M., Haroche, S.: Observation of cavity-enhanced singleatom spontaneous emission. Phys. Rev. Lett. 50 1903-1906 (1983)

38. Purcell, E. M.: Spontaneous emission probabilities at radio frequencies. Phys. Rev. 69 681-685 (1946)

39. Wang, Q., Liao, J. Q., Zeng, H. S.: Quantum thermal discord in a two-spin-1/2 XXZ model. Chin. Phys. B 19100311 (pp.1-5) (2010)

40. Mogilevtsev, D., Nisovtsev, A. P., Kilin, S., Cavalcanti, S. B., Brandi, H. S., Oliveira, L. E.: Driving-dependent damping of Rabi oscillations in two-level semiconductor systems. Phys. Rev. Lett. 100017401 (pp.1-4) (2008)

41. Dubin, F., Rotter, D., Mukherjee, M., Russo, C., Eschner, J., Blatt, R.: Photon correlation versus interference of single-atom fluorescence in a half-cavity. Phys. Rev. Lett. 98 183003 (pp.1-4) (2007)

42. Reitzenstein, S., Hofmann, C., Gorbunov, A., Strauss, M., Kwon, S. H., Schneider, C., Löffler, A., Höfling, S., Forchel, A.: AlAs/GaAs micropillar cavities with quality factors exceeding 150.000. Appl. Phys. Lett. 90251109 (pp.1-3) (2007)

43. Bayer, M., Forchel, A.: Temperature dependence of the exciton homogeneous linewidth in $\mathrm{In}_{0.60} \mathrm{Ga}_{0.40} \mathrm{As} / \mathrm{GaAs}$ self-assembled quantum dots. Phys. Rev. B 65041308 (R) (pp.14) (2002) 